\title{
UNA MODALITÀ DI REALIZZAZIONE DEL SERVICE LEARNING: IL PROGRAMMA MENTOR-UP
}

Giada Tomelleri, Massimo Santinello, Marisa Bergamin, Claudia Marino ${ }^{60}$

\section{Sintesi}

Nell'articolo, dopo una breve introduzione sul modello del mentoring con i minori a rischio, viene presentata una sintetica rassegna della letteratura sulle connessioni tra mentoring e service learning e successivamente su quali processi attiva una relazione di mentoring.

Parole chiave: Mentoring. Service Learning. Minori a rischio. Mentor-UP.

\section{UMA MODALIDADE DE REALIZAR A APRENDIZAGEM SOLIDÁRIA: O PROGRAMA MENTOR-UP}

\section{Resumo}

No artigo, após uma breve introdução ao modelo de orientação com menores em risco, é apresentada uma breve revisão da literatura sobre as conexões entre mentoria e aprendizagem solidária e, posteriormente, sobre quais processos são ativados por um relacionamento de mentoria.

Palavras-chave: Mentoria. Aprendizagem solidária. Menores em risco. Mentor-UP.

\section{A WAY OF REALISATION OF SERVICE LEARNING: THE MENTOR-UP PROGRAM}

\begin{abstract}
In the article, after a brief introduction on the model of mentoring with minors at risk, a brief review of the literature is presented on the connections between mentoring and service learning and subsequently on which processes activate a mentoring relationship.
\end{abstract}

Keywords: Mentoring. Service learning. Minors at risk. Mentor-UP.

${ }^{60}$ Dipartimento di Psicologia dello Sviluppo e della Socializzazione - Università di Padova 
Mentor-UP è un'esperienza che permette agli studenti di apprendere nuove competenze e che risponde a un bisogno della comunità locale. In generale, con mentoring ci «si riferisce a una relazione tra un minore (Mentee) e una persona più adulta (Mentore), che non ha legami familiari con lui» (Dolan \& Brady, 2012, p. 10). L’Università di Padova utilizza questo modello per favorire l'acquisizione di competenze relazionali, sviluppare senso civico e favorire lo sviluppo positivo di ragazzi a rischio di età compresa tra i 9 e i 12 anni.

\section{Le ragioni di un programma di mentoring}

Negli ultimi decenni si sono molto ridotte le occasioni di sostegno informale ai minori da parte degli adulti, sia familiari che di altri soggetti al di fuori delle reti sociali primarie (Dolan \& Brady, 2012). Questo fenomeno è dovuto al cambiamento delle dinamiche e delle forme familiari, all'aumento da parte dei genitori del tempo dedicato al lavoro retribuito, alla maggiore incertezza e "liquidità" che caratterizza la società contemporanea e, di conseguenza, a minori opportunità per i giovani di poter fruire di una figura stabile e sicura da prendere come modello per uno sviluppo sano ed equilibrato.

Tendenzialmente i programmi di mentoring si rivolgono soprattutto ai giovani che si trovano nelle fasce d'età caratterizzate dal cambiamento, quali sono la preadolescenza e l'adolescenza. In questo senso, la presenza di un Mentor nella vita di un minore può fungere da fattore protettivo in un momento dello sviluppo critico e può aiutare il Mentee ad affrontare situazioni potenzialmente stressanti (Dolan \& Brady, 2012). Inoltre, la capacità di un preadolescente/adolescente di fronteggiare eventi stressanti dipende dalla percezione di disponibilità di trovare occasioni di sostegno sociale da parte di adulti significativi (Bal, Crombez, Van Oost, \& Debourdeaudhuij, 2003).

Un'ulteriore ragione che dà significato ad un programma di mentoring è la constatazione che i ragazzi resilienti, ossia quelli che sono stati in grado di fronteggiare le avversità nel corso del loro sviluppo, sono ricorsi all'aiuto di un adulto significativo, ossia un Mentor, sia esso formale o informale (Dolan \& Brady, 2012). Quindi la partecipazione a programmi di mentoring formale può aiutare il minore a sviluppare quelle competenze e risorse personali e sociali necessarie per affrontare le sfide quotidiane che gli si paleseranno nel corso della vita.

Infine, i programmi strutturati di mentoring offrono ai minori la possibilità di creare una relazione di fiducia con un adulto, di ampliare la rete sociale e la conoscenza 
delle opportunità offerte dal territorio; aiutano il minore a stabilire delle forti connessioni sociali tra più individui e istituzioni, per consentirgli sia di avere uno sviluppo positivo in adolescenza sia di transitare con successo nel mondo adulto (Eccles \& Gootman, 2002).

\section{2: Il mentoring e il service learning}

Alcuni studi si sono posti l'obiettivo di capire in che modo partecipare a un programma di mentoring attraverso la modalità di service learning portasse dei benefici agli studenti. Secondo lo studio di Weiler e colleghi (Weiler, Haddock et al., 2013) offrendo agli studenti universitari l'opportunità di essere Mentor, si permette loro di diventare persone dotate di senso civico, supportando lo sviluppo di giovani a rischio nel loro territorio. Inoltre, il loro servizio può essere utile a ridurre il carico di responsabilità delle agenzie di servizi per la comunità, spesso tese a rispondere alle esigenze di un grande numero di utenti. Infine, poiché i Mentor coinvolti nel programma risultano propensi a rimanere coinvolti nella loro comunità, quest'ultima acquisisce cittadini impegnati e qualificati. I risultati hanno inoltre individuato un maggiore impegno da parte degli studenti nella partecipazione civica, una migliore conoscenza dello sviluppo adolescenziale e un maggior orientamento a obiettivi legati all'istruzione/educazione. In una ricerca successiva (Moses, Fry, \& Sanders, 2014) è emerso che la partecipazione di studenti in attività di service learning porta ad acquisire una maggiore consapevolezza della diversità e della complessità delle vite dei loro Mentee. Inoltre, molti di loro vivono questa esperienza come una sfida e ciò aggiunge valore al loro apprendimento accademico. In terzo luogo indicano, tra le abilità acquisite, un miglioramento della comunicazione, dell'ascolto e della capacità di offrire consigli non giudicanti.

Inoltre, il mentoring con i minori consente di conoscere in modo più approfondito il background familiare, sociale ed economico da cui provengono i minori, nonché le diversità della comunità in cui loro stessi vivono (Moses, Fry, \& Sanders, 2014).

Nello studio di Kafai et al. (2008) gli autori hanno raccolto oltre 200 diari nei quali, quasi la maggior parte dei Mentor, descrivono l'esperienza come occasione di apprendimento oltre che di riflessione sulle problematiche sociali. La diade è assunta a partnership coinvolta alla pari nel processo di apprendimento. Fresko \& Wertheim (2006) riscontrarono che l'esperienza di mentoring con ragazzini a rischio risultava più significativa per gli studenti interessati a proseguire la carriera come insegnanti, soprattutto dal punto di vista dell'incremento della sensibilità nei confronti di ragazzini 
provenienti da contesti sociali e culturali differenti. Per gli studenti di Psicologia, Servizio sociale e Medicina l'esperienza di mentoring ha contribuito a sviluppare la capacità di empatia e l'interesse verso gli altri.

Hughes, C., Welsh, M., Mayer, A., Bolay, J., \& Southard, K. (2009) hanno investigato gli effetti di un programma nel quale gli studenti dell'università affiancavano giovani delle scuole superiori appartenenti ad un contesto di alta povertà. Ai Mentor venne chiesto prima dell' inizio del programma di riflettere sulle proprie aspettative, e alla conclusione del programma, di riportare la percezione del loro conseguimento. Le aspettative riguardavano il desiderio di stabilire una relazione di amicizia con i Mentee, piuttosto che di tutoring scolastico, e di poter conoscere gli effetti della povertà. Generalmente tali aspettative sono state ritenute conseguite al termine dell'esperienza.

\section{Quali meccanismi sono implicati nella relazione di mentoring}

Il modello di mentoring sviluppato da Rhodes nel 2005, individua tre processi di sviluppo che, interagendo tra loro e grazie a una relazione di mentoring solida, permettono il raggiungimento di risultati positivi (D'Alessio, Laghi, \& Giacalone, 2010). I tre processi in questione sono lo sviluppo socio-emotivo, lo sviluppo cognitivo e la costruzione dell'identità.

Per quanto riguarda il primo, l'instaurarsi di una relazione solida e significativa tra un Mentor e un Mentee può portare i minori a migliorare la loro percezione del sostegno sociale e facilitare così una positiva connessione con gli altri (Raposa, et al., 2019). La percezione dei ragazzi del sostegno ricevuto da parte del Mentor è risultato avere la stessa importanza del sostegno effettivamente ricevuto, il che porta a pensare che la mera esistenza di una relazione positiva con il Mentor può portare nei minori la percezione di un miglioramento a vantaggio della loro crescita personale (Dolan \& Brady, 2012); inoltre, il fatto di vivere esperienze positive dal punto di vista socio-emotivo con il Mentor permette ai minori di sviluppare modalità di interazione con gli altri più efficaci ed equilibrate (D'Alessio, Laghi, \& Giacalone, 2010). L'influenza che la relazione di mentoring può avere sulle precedenti e successive interazioni sociali è rimandabile alla teoria dei legami di attaccamento di Bowlby. Attraverso la teoria dell'attaccamento, l'autore spiega le «differenze individuali di bambini e adolescenti nell'interazione con gli altri significativi, ossia i genitori, il gruppo dei pari e i partners» (Noam, Malti, \& Karcher, 2014, p. 100). Il legame di attaccamento si forma in tenera età a partire dal 
rapporto con i genitori o con una figura di riferimento e generalmente rimane stabile nel corso dello sviluppo, anche se può subire delle variazioni in base a circostanze future, in questo caso la formazione di una relazione di mentoring positiva; ciò significa che vi è una relazione biunivoca tra $\mathrm{i}$ modelli di attaccamento che si sono formati durante l'infanzia e le successive esperienze relazionali con adulti significativi. Questo concetto risulta particolarmente evidente nel caso in cui il Mentee abbia instaurato con il caregiver un modello di attaccamento ansioso-ambivalente o ansioso-evitante. Nel primo caso il minore fatica a porre fiducia nell'idea che gli altri possano prendersi cura di lui nel lungo termine, preoccupazione che lo spinge a chiedere frequentemente rassicurazioni da parte delle persone. Nel secondo caso, il minore tenderebbe a evitare forme di relazione stabili e intime con gli altri a causa di una sfiducia nei loro confronti (Noam, Malti, \& Karcher, 2014). La relazione di Mentoring si potrà quindi ritenere significativa nella misura in cui riuscirà a modificare i modelli operativi interni disfunzionali dei minori, offrendo loro uno schema di relazione positivo e sano da riprodurre nelle successive interazioni sociali.

Il secondo processo, quello relativo allo sviluppo cognitivo, fa riferimento al ruolo del sostegno sociale da parte di adulti nel favorire l'acquisizione di abilità cognitive (Rhodes \& DuBois, 2008). In particolare, questo processo è favorito e sostenuto dal coinvolgimento dei minori in attività e in conversazioni significative con adulti capaci di generare idee originali, di comprendere e analizzare i concetti in profondità e di utilizzare un pensiero logico nella risoluzione di problemi complicati (Raposa, et al., 2019). Inoltre, attraverso l'interazione con i Mentor, i bambini e gli adolescenti hanno la possibilità di acquisire e affinare nuove modalità di pensiero e di rendersi più ricettivi rispetto ai valori, ai consigli e alle prospettive degli adulti (Rhodes \& DuBois, 2008). Nel corso dello sviluppo i minori vivono dei cambiamenti nel modo di percepire e comprendere se stessi e le relazioni con gli altri, perciò il processo di sviluppo socio-cognitivo è il risultato dell'interazione continua che il soggetto ha con l'esterno e la maturazione biologica del sistema cognitivo (Noam, Malti, \& Karcher, 2014, p. 103). Durante l'età preadolescenziale i minori acquisiscono la relatività dei punti di vista, ossia riconoscono che le intenzioni e le prospettive proprie e altrui possono essere diverse e in quanto tali comprese e negoziate. In una relazione di mentoring risulta importante conoscere $\mathrm{i}$ cambiamenti che nascono in un preadolescente poiché ciò permette al Mentor di valutare quanto e come il suo Mentee è in grado di assumere la sua prospettiva e di riconoscere le proprie intenzioni, emozioni, bisogni e desideri, sia in generale per quanto riguarda la sua vita, sia in particolare nella relazione di mentoring: un minore che non ha raggiunto la 
maturità cognitiva adatta a permettergli di coordinare molteplici punti di vista (in questo caso il suo e quello del suo Mentor) sarà meno capace di cogliere il significato di certe attività o conversazioni che il Mentor gli propone (Noam, Malti, \& Karcher, 2014). L'incapacità dei Mentee di cogliere la prospettiva dei Mentor può derivare da una normale limitazione cognitiva che dovrebbe portare i Mentor a guidare e supportare i minori nello sviluppo della capacità di perspective taking; la relazione di mentoring in questo senso funge da contesto protetto e sicuro in cui i minori possono allenare delle abilità cognitive che ancora non mostrano nel presente ma che potrebbero padroneggiare in futuro se supportati e incoraggiati. Questo aspetto richiama il concetto di zona di sviluppo prossimale di Vygotskij, ossia quell'area di apprendimento potenziale in cui può collocarsi un bambino grazie all'aiuto di un adulto o di un coetaneo più esperto. L'adulto, in questo caso il Mentor, attraverso lo scaffolding, fungerebbe da "impalcatura" $\mathrm{e}$ supporto nel processo di scoperta da parte del Mentee del suo potenziale di sviluppo che altrimenti rimarrebbe inesplorato. Questo ruolo di supporto da parte del Mentor risulta particolarmente efficace nella vita scolastica del Mentee, quando quest'ultimo ha la percezione di non possedere determinate abilità $\mathrm{o}$, pur possedendole, non si ritiene in grado di utilizzarle. Quindi, in questo ambito, la relazione di mentoring può «incidere sull'aspetto motivazionale e volitivo dello studente» (D'Alessio, Laghi, \& Giacalone, 2010, p. 78) e potenziare così gli aspetti metacognitivi dei minori. Con metacognizione si intende la conoscenza del proprio e altrui funzionamento cognitivo e l'utilizzo di strumenti di controllo per monitorare la propria attività cognitiva (D'Alessio, Laghi, \& Giacalone, 2010). In ambito scolastico, compito del Mentor è quello di aiutare i Mentee a diventare consapevoli delle loro potenzialità, aiutandoli a incrementare il loro senso di autoefficacia scolastica. L'autoefficacia si struttura a partire da molteplici fonti: le esperienze personali, ossia il fatto di vivere in prima persona esperienze di padronanza di una situazione (quale può essere un compito scolastico); le esperienze vicarie, ossia attraverso l'osservazione di un modello positivo di comportamento (quale può essere il Mentor); la persuasione verbale, quindi l'incoraggiamento e la fiducia dimostrata da persone significative in un momento di difficoltà nell' affrontare una situazione stressante; gli stati emotivi e fisiologici, ossia la percezione della propria attivazione fisiologica ed emotiva e le modalità utilizzate per controllare e gestire l'attivazione stessa. Dal momento che il sostegno sociale e la formazione di relazioni intime e positive possono incrementare il successo accademico, è evidente quanto il minore possa trovare giovamento da una relazione di mentoring basata sulla fiducia e sull'incoraggiamento. 
Il terzo processo preso in considerazione è quello relativo allo sviluppo dell'identità, in cui il Mentor riveste un ruolo importante perché funge da modello positivo che i Mentee possono emulare e offre ai ragazzi nuovi contesti e risorse per esplorare i loro interessi (Raposa, et al., 2019). Lo sviluppo dell'identità è un processo che attraversa le fasi dell'intero ciclo di vita, in cui gli individui sono chiamati ad affrontare e superare specifici compiti evolutivi per poter transitare nella fase successiva; è però durante l'età preadolescenziale e adolescenziale che i minori iniziano a orientare il proprio sviluppo verso un determinato modello o percorso, sulla base dei loro personali valori, interessi e capacità. Ad esempio nella seconda metà dell'infanzia e nella preadolescenza (indicativamente dai 6 ai 12 anni), il minore inizia a identificare le attività che lo interessano e da cui trae giovamento e ciò gli è utile per sviluppare la padronanza di certe abilità piuttosto di altre (Noam, Malti, \& Karcher, 2014). Soprattutto durante l'età scolare i bambini hanno la necessità di affermare se stessi e ricevere approvazione dall'esterno; ciò li porta a orientarsi verso attività e compiti che permettono di sviluppare nuove abilità. Durante la prima adolescenza (dai 10 ai 14 anni circa), il minore ha bisogno di esplorare se stesso e capire la percezione che gli altri hanno di lui. Nella fase della media adolescenza, invece, il compito principale è quello di integrare gli interessi, i ruoli e le molteplici percezioni di se stessi in una concezione di sé globale e di percepire un senso di appartenenza al gruppo dei pari. Queste informazioni sono importanti per il Mentor perché gli permettono di capire dove orientare il focus della relazione ed è importante che le conversazioni e le attività da lui proposte siano finalizzate a soddisfare ciò che è richiesto nelle specifiche età (Noam, Malti, \& Karcher, 2014). Il Mentor dovrebbe quindi «valutare in che modo ogni interazione con il Mentee può promuovere iniziative di supporto per il suo sviluppo [...] quando si presentano opportunità per poterlo fare all'interno del programma di Mentoring» (Noam, Malti, \& Karcher, 2014, p. 111). In questo modo l'adulto può aiutare il minore ad affrontare con successo i compiti evolutivi dell'età in cui si trova ed empatizzando con i suoi bisogni è più probabile che si rinforzi la relazione di mentoring.

I tre processi descritti (socio-emotivo, cognitivo e costruzione dell'identità) sono interconnessi tra loro e la crescita in alcune abilità cognitive può aiutare i minori a comprendere e regolare le emozioni più complicate; inoltre, un Mentor che funge da modello per lo sviluppo può aiutare il Mentee a promuovere la sua capacità di stringere legami intimi e sicuri e di capire il mondo da una prospettiva diversa dalla propria. Per 
questo la relazione di Mentoring permette di riscontrare dei risultati positivi in molteplici ambiti della vita di un giovane (Raposa, et al., 2019).

\section{Bibliografia}

Bal, S., Crombez, G., Van Oost, P., \& Debourdeaudhuij, I. (2003). The role of social support in well-being and coping with self-reported stressful events in adolescents. Child Abuse \& Neglect, 27(12), 1377-1395.

D'Alessio, M., Laghi, F., \& Giacalone, V. (2010). Quali dimensioni indagare. In M. D'Alessio, F. Laghi, \& V. Giacalone, Mentoring e scuola: Teorie, modelli e metodologie di intervento a contrasto della dispersione scolastica. (p. 73-100). Milano: Ulrico Hoepli.

Dolan, P., \& Brady, B. (2012). Il Mentoring con i minori: strategie e modelli di intervento. Trento: Edizioni Erickson.

Eccles, J., \& Gootman, J. A. (2002). Community Programs to Promote Youth Development. Washington, DC: National Academy Press.

Fresko, B., Wertheim, C. (2006). Learning by mentoring: Prospective teachers as mentors to children at-risk, Mentoring \& Tutoring: Partnership in Learning, 14 (2), 149-161.

Hughes, C., Welsh, M., Mayer, A., Bolay, J., Southard, K. (2009). An innovative university based mentoring program: Affecting college students' attitudes and engagement. Michigan Journal of Community Service Learning, Fall, 69-78.

Kafai, Y. B., Desal, S., Peppler, K. A., Chiu, G. M., Moya, J. (2008). Mentoring partnerships in a community technology centre: A constructionist approach for fostering equitable service learning. Mentoring \& Tutoring: Partnership in Learning, 16 (2), 191204.

Moses, W., Fry, J., \& Sanders, K. (2014). The Impact of a Service-Learning Experience in Mentoring At-Risk Youth. Journal on Excellence in College Teaching., 71-94.

Noam, G. G., Malti, T., \& Karcher, M. J. (2014). Mentoring Relatioships in Developmental Perspective. In D. L. DuBois, \& M. J. Karcher, Handbook of Youth Mentoring. (p. 99-115). Thousand Oaks, California USA: SAGE Publications.

Raposa, E.B., Rhodes, J.E., Stams, G.J.J.M., Card, N., Burton. S., Schwartz, S., Sykes, L.A., Kanchewa, S., Kupersmidt, J., Hussain, S. (2019). The effects of youth mentoring programs: A meta-analysis of outcome studies. Journal of Youth and Adolescence, 48, doi: 10.1007/s10964-019-00982-8.

Rhodes, J.E. \& DuBois, D.L. (2008). Mentoring relationships and programs for youth. Current Directions in Psychological Science, 17, 254-258.

Weiler, L., Haddock, S., Zimmerman, T.S., Krafchick, J., Henry, K., Rudisill, S. (2013). Benefits derived by college students from mentoring at-risk youth in a Service-learning course. American Journal of Community Psychology, 52, 236-248. 